\title{
A educação como instrumento de mudança na prestação de cuidados para idosos
}

\section{Education as an instrument of change in the provision of care for the elderly}

\author{
Henrique Salmazo da Silva* \\ Beatriz Aparecida Ozello Gutierrez ${ }^{* *}$
}

\begin{abstract}
RESUMO
A Instituição de Longa Permanência do Idoso (ILPI) caracteriza-se como uma modalidade de atenção importante no cenário dos cuidados de longa duração. No Brasil urge a necessidade de refletirmos sobre o aparato técnico e humano que subsidia o funcionamento desses serviços, em especial pela complexidade de saúde dos residentes. Considerando essas questões, o objetivo deste artigo foi discutir a educação como eixo norteador para a melhoria dos cuidados prestados nas ILPI, destacando o cuidar como um processo atitudinal que revela a contínua interação entre conhecimentos técnicos e a humanização, bem como a valorização da opinião da pessoa idosa sobre diferentes aspectos do cotidiano institucional. Para tanto, foi realizado levantamento bibliográfico de periódicos e artigos publicados nos últimos dez anos, utilizando as bases de dados da PUBMED e SCIELO. Verificou-se que as intervenções educativas podem oferecer ferramentas importantes para a melhoria dos cuidados, fomentando mudanças organizações, a adoção de novas tecnologias de cuidado e a criação de legislações compatíveis com as necessidades dos idosos assistidos.
\end{abstract}

Palavras-chave: Idoso. Educação. Cuidados. Institucionalização. Intervenções. Saúde.

* Universidade Católica de Brasília, Brasília, Distrito Federal, Brasil. E-mail: henriquesalmazo@yahoo.com.br. http://orcid.org/0000-0002-3888-4214

** Escola de Artes, Ciências e Humanidades da Universidade de São Paulo. São Paulo, São Paulo, Brasil. E-mail: biaagutierrez@gmail.com. http://orcid.org/0000-0001-6901-6439 


\begin{abstract}
The nursing home is characterized as a type of important attention in the setting of long-term care. In Brazil there is an urgent needing to reflect on the human and technical apparatus which subsidizes these services, specially taking in consideration the health complexity of residents. Considering these issues, the aim of this article was to discuss education as a guideline for the improvement of care in nursing homes, highlighting care as an attitudinal process that shows the continuous interaction between technical knowledge and humanization, as well as the appreciation of view elderly about different aspects of the institutional routine. For this we carried literature review in journals and articles published in the last ten years, using the databases PUBMED and SCIELO. It was found that educational interventions can provide important tools for improving care, promoting change organizations, the adoption of new care technologies and the creation of legislation compatible with the needs of the older adults assisted.
\end{abstract}

Keywords: Elderly. Education. Care. Institutionalization. Interventions. Health.

\title{
Introdução
}

Alcançar idades avançadas e estar amparado por uma rede de cuidados é um dos desafios mais expressivos de estudiosos e gestores públicos. As mudanças no cenário de saúde pública observadas nas últimas décadas contribuíram para a elevação da expectativa de vida da população idosa, com aumento expressivo dos idosos que alcançam a quarta idade (75 anos e mais) e dos centenários, que já totalizam 23.760 brasileiros (CAMARANO; KANSO, 2011). Embora, a população dos mais longevos cresça em ritmo acelerado, os estudos populacionais indicam que são esses idosos que requerem maior investimento de cuidados de longa duração e de políticas públicas que visem um cuidado qualificado, uma vez que possuem mais chances de viver sozinhos (CAMARANO, KANSO, 2011), de ter mais dependência (CAMARANO, KANSO, 2011) e complexidade assistencial (GUTIERREZ; SALMAZO-SILVA; SHIMIZU, 2014) e de reportarem menor suporte social em comparação aos idosos mais jovens (FONTES et al., 2011).

Os cuidados de longa duração compreendem uma variedade de serviços médicos, sociais e de atenção com o objetivo de auxiliar os indivíduos a viverem de forma mais independente possível, maximizando os seus níveis de funcionamento físico e psicológico (FERRINI; FERRINI, 2008). Esses cuidados são prestados em vários âmbitos: na atenção primária de saúde, nos domicílios 
(cuidados domiciliares), centros de convivência, centros dia e nas Instituições de Longa Permanência para Idosos (ILPIs) visando oferecer apoio às famílias e aos idosos situados em diferentes graus de dependência e vulnerabilidade social.

Atualmente, pela escassez de cuidados domiciliares e de programas brasileiros que maximizem a permanência do idoso no domicílio, uma das alternativas são as ILPIs que constituem uma modalidade importante de cuidados fora do domicílio.

A ILPI segundo a Agência Nacional de Vigilância Sanitária (ANVISA, 2005) na Resolução da Diretoria Colegiada (RDC) n 283, é uma instituição governamental ou não governamental, de natureza residencial, destinada à moradia coletiva de indivíduos com idade igual ou superior a 60 anos, com ou sem suporte familiar, em condições de liberdade, dignidade e cidadania. Estabelece ainda que, a ILPI deve ser um lar especializado, com dupla função - de proporcionar assistência gerontogeriátrica conforme o grau de dependência dos seus residentes e de oferecer, ao mesmo tempo, um ambiente doméstico, aconchegante, capaz de preservar a intimidade e a identidade dos seus residentes.

No contexto dos cuidados de longa duração, o cuidado ao idoso institucionalizado no Brasil suscita inúmeros desafios, considerando que colocar um familiar ou um ente querido em uma instituição ainda é um tabu, principalmente porque culturalmente os filhos foram incumbidos da missão de cuidar de seus pais. Também, o próprio Estatuto do Idoso, promulgado em 2003, reforça a responsabilidade da família no cuidado ao idoso. Embora, a família seja a principal provedora de cuidados, nem sempre possui o aparato necessário para cuidar dos seus idosos no domicílio de forma intensiva, sendo necessário institucionalizá-los.

Além dos desafios mencionados, destacam-se ainda: o idoso institucionalizado tem uma complexidade assistencial de saúde que solicita maior demanda de cuidados; as ILPIs brasileiras públicas estão vinculadas ao Sistema Único de Assistência Social (SUAS) e respaldadas pelo Sistema Único de Saúde (SUS) que, às vezes, é insuficiente para suprir todas as necessidades de saúde dessa população; e ainda, os recursos estabelecidos pela legislação vigente relacionados às normas técnicas de funcionamento das ILPIs, algumas vezes, apresentam-se inviáveis com a realidade que o gestor enfrenta no seu dia a dia de trabalho, pois os parâmetros mínimos instituídos são incompatíveis com a demanda dos serviços.

Diante dessa problemática, a Organização Mundial de Saúde (OMS) junto à Associação Internacional de Geriatria e Gerontologia na Advancing Nursing Home Practice, em 2014, reuniram pesquisadores e profissionais com o objetivo de levantar ações e estratégias visando melhorias na qualidade assistencial das ILPIs, entre as quais, destaca-se a humanização dos cuidados prestados aos 
idosos institucionalizados, considerando todos os atores envolvidos: os clientes, equipe profissional, a rede assistencial, as políticas institucionais, as políticas governamentais e a relação das práticas assistenciais com indicadores objetivos e subjetivos de saúde, como a qualidade de vida (TOLSON; ROLLAND; ANDRIEU, 2011; MORLEY et al., 2014). Embora, os cuidados estejam mais avançados do ponto de vista técnico nos países desenvolvidos, a OMS salientou que mesmo nesses países os cuidados institucionais ainda precisam de um esforço extra, advindo de diferentes áreas da Gerontologia e das ciências da saúde. Para avançar torna-se vital a práxis, articulando a teoria e a prática com conhecimento detalhado a respeito do que os residentes consideram como cuidados dignos e de qualidade e o amadurecimento de preceitos éticos e culturais que perpassam as atitudes da equipe para com as necessidades desses residentes.

Com base nessas questões, os objetivos deste artigo é discutir ferramentas inovadoras para o cuidado centrado no indivíduo, focalizando a opinião da pessoa idosa sobre diversos aspectos do cotidiano institucional e propor a educação como eixo norteador para a melhoria dos cuidados nas ILPIs, destacando o cuidar como um processo atitudinal produto da interação entre técnica e humanização. A presente discussão se baseou em revisão da literatura utilizando a base de dados da PUBMED de 2005 a 2015. Utilizamos os descritores: "nursing home and quality of life" e "nursing home and care". Os estudos tratam sobre os cuidados institucionais e a qualidade de vida dos residentes permeados pela comunicação.

\section{Ferramentas de comunicação e escuta para o bem-estar dos residentes}

Entendemos neste estudo que ferramentas são veículos utilizados nos processos de trabalho que visam à sistematização, à operacionalização e à hierarquização de procedimentos e necessidades para se atingir os objetivos propostos. Entre essas ferramentas destacamos a importância da comunicação e da escuta, sendo um processo de contínua interação entre profissionais e residentes, focalizando a percepção subjetiva do que o residente considera como importante para sua vida e para o seu bem-estar.

Consideramos que o crescimento técnico e humano da equipe de trabalho ultrapasse as fronteiras que delimitam o distanciamento nas relações de cuidado guiadas somente por procedimentos. Nesse sentido, o enfoque passa a ser além do tratamento de saúde, pois o profissional deve acolher o idoso, possibilitando que floresçam subsídios para o enfrentamento de possíveis dificuldades que possam estar presentes nessa sua fase da vida. 
Os achados da literatura indicam que o processo de escuta está associado a uma série de indicadores de bem-estar dos residentes. Entre eles destacam o empoderamento, caracterizado pela capacidade de reconhecer, promover e aumentar a habilidade dos pacientes em identificar suas necessidades e, sentir o controle sobre suas vidas sobre os cuidados esteve associado à qualidade de vida dos residentes (TU; WANG; YEH, 2006). Também, medidas de bem-estar psicológico, propósito na vida, crescimento pessoal estão atrelados à função da autonomia dos residentes e ao senso de que suas necessidades estão sendo atendidas (FERRAND et al., 2014). Em outra direção, criticismo e hostilidade estiveram associados aos sintomas de estafa presentes nos profissionais das ILPIs (TANAKA, 2015).

Em virtude da importância desses indicadores, os cuidados institucionais devem incluir itens como segurança, conforto, número de atividades significativas, qualidade das relações sociais, dignidade, autonomia, privacidade, individualidade, bem-estar espiritual e competência funcional (KANE, 2001). Nessa abordagem, o enfoque nas necessidades dos residentes parece ser a chave para a criação de programas que tornem esses ambientes mais humanizados, acessíveis e acolhedores (PUTNAM et al., 2007).

Pensando assim, para alcançar esse objetivo algumas instituições criaram protocolos de atendimento com informações sobre as preferências de atendimento nos quartos e de convivência institucional com base nos valores e crenças dos atendidos; a elaboração de rotinas que almejam extinguir o ócio não criativo e a solidão e o desenvolvimento de novas tecnologias para melhorar a acessibilidade e o conforto dos idosos com diferentes graus de funcionalidade. Dessa forma, o desenvolvimento de novas tecnologias pode auxiliar tanto os idosos quanto a equipe de trabalho dessas instituições, aumentando o senso de competência e a interação entre assistidos e equipe profissional (MORLEY et al., 2014).

Também, modelos de cuidados e propostas de intervenção são sugeridos para diminuir a experiência de solidão e a perda da autonomia (BROWNIE; HORSTMANSHOF, 2011; EKMAN et al., 2011). A solidão foi definida como sentimento incômodo que resulta da discrepância entre as relações sociais atuais e as desejáveis (BROWNIE, HORSTMANSHOF, 2011). A causa desse sentimento no cotidiano institucional pode ser consequência de condições de saúde (demências, declínio funcional), do empobrecimento da rede social (viuvez, distanciamento da rede social) ou do desengajamento nas atividades pregressas (BROWNIE; HORSTMANSHOF, 2011; THEURER et al., 2015). Estima-se que $55 \%$ dos idosos institucionalizados vivenciem essa experiência e sabemos pouco sobre quais idosos são mais suscetíveis e como intervir (BROWNIE; HORSTMANSHOF, 2011; THEURER et al., 2015). 
Nessa abordagem, torna-se relevante que os profissionais realizem um estudo profundo da história pregressa individual dos idosos e, adicionalmente acrescente intervenções nas ILPIs que observem os preceitos teóricos da produtividade e identidade social (THEURER et al., 2015). Assim, a produtividade social pode ser considerada como uma forma de troca interpessoal que se ancora na noção de reciprocidade (intercâmbio entre o dar e receber) (THEURER et al., 2015). A identidade social, por sua vez, baseia-se na partilha do que temos em comum. Nesse construto, mesmo para os idosos com demência e capacidade funcional reduzida, as atividades devem ser contextualizadas, respeitando as limitações do idoso, sua identidade e a sua maneira de Ser que contribui e que compartilha.

No que se refere à capacidade funcional dos residentes, por meio de uma revisão sistemática sobre as intervenções institucionais de 1990 a 2015, foram observadas intervenções que visam aumentar a funcionalidade dos residentes diminuem a sobrecarga da equipe, mas o contrário não foi observado, isto é, intervir nos profissionais para gerar benefícios indiretos nos residentes (LOW et al. 2015). Outro resultado observado foi que mudanças nos cuidados diretos (alimentação, higiene, protocolos de prevenção de quedas, por exemplo) geraram impactos mais robustos do que intervenções gerais, cuja eficácia poderia solicitar o planejamento conjunto de intervenções menores (por exemplo, protocolos sobre a qualidade dos cuidados nas demências) (LOW et al. 2015).

Gestores dos equipamentos elencaram uma série de ações para tornar os cuidados mais flexíveis às necessidades dos residentes, solicitando mudanças estruturais e organizacionais dos cuidados (CORNALLY et al., 2015), incluindo: documentação, levantamento de recursos externos, inclusão desses conceitos na admissão de novos residentes e implementação de processos educativos e capacitação da equipe.

\section{Intervenções institucionais subsidiadas por processos educativos}

Estudos focalizaram intervenções educativas abordando alguns temas sugeridos pelos especialistas da OMS, organizando o alvo dos processos educativos em eixos como: revisão da medicação, processos de avaliação da dor e tratamento, habilidades de comunicação e organização de atividades; sendo esses eixos ancorados pelo princípio de segurança (HUSEBO, 2015).

A adoção de intervenções educativas aliada às mudanças na cultura organizacional desses espaços seriam as principais frentes para as intervenções institucionais nas ILPIs, gerando resultados promissores tanto na prevenção de 
desfechos institucionais negativos como a violência, a alta rotatividade e adoecimento de profissionais, quanto na melhoria da qualidade de vida de todos os atores envolvidos (TOLSON D.; ROLLAND Y; ANDRIEU, 2011; MORLEY, et al. 2014; BORN; BOECHAT, 2011; CAMARANO et al., 2010; ALCÂNTARA, 2009; CORTELLETTI; CASARA; HERÉDIA, 2010).

Em revisão de literatura, (COOPER et al., 2008) identificaram as seguintes estimativas de violência dentro de instituições: $16 \%$ dos profissionais relataram cometer abuso psicológico significativo; $10 \%$ admitiram abuso físico e $40 \%$ de qualquer ato psicologicamente agressivo no intervalo de um ano; e mais de $80 \%$ referiram ter observado abusos. É possível que essas estimativas estejam associadas às crenças desses profissionais, à falta de motivação, pouca remuneração e baixo conhecimento técnico sobre idoso e Gerontologia. Neri (2006) ressaltou que profissionais de ILPIs que apresentavam crenças negativas sobre o processo de envelhecimento tiveram maior insatisfação no cotidiano profissional, negligenciando, muitas vezes, procedimentos de analgesia para amenizar a dor dos pacientes. A escassez de informações, somada à insatisfação profissional, levava os trabalhadores a cometerem violência velada, cuja notificação e intervenção eram de difícil acesso. Estudos indicaram que esses profissionais se orientam para os requisitos básicos como higiene e alimentação e tendem a reproduzir um modelo de cuidado que enfatiza as perdas e a dependência (CREUTZBERG et al., 2007; BALTES, 1996; PAVARINI, 1996).

Em pesquisa conduzida por Baltes (1996) identificou-se que o padrão esperado pelos idosos e profissionais é o de declínio e redução do desempenho funcional, levando-os a expectativas cada vez menores sobre o potencial de funcionamento que poderiam ter. No entanto, em serviços institucionais direcionados para crianças observou-se o contrário, pois os profissionais estimularam mais a independência por acreditarem que o desenvolvimento infantil, diferente da velhice, implica mais ganhos do que perdas.

Nesse contexto, o desafio às organizações se traduz em proporcionar condições para que existam melhorias nas competências profissionais da sua equipe para que tenham a possibilidade de aperfeiçoar o seu conhecimento, a sua habilidade e a sua atitude (BRANDÃO; GUIMARÃES, 2001). Assim, essa equipe terá condições de: adquirir conhecimentos na área da geriatria e gerontologia; em relação à habilidade, conseguir reproduzir modelos de cuidados baseados na escuta e no crescimento humano, focalizando as potencialidades do residente, no sentido de favorecer o seu empoderamento e priorizar a sua autonomia diante das relações de cuidados que são produzidas e ainda, no que se refere às atitudes imperar o respeito e a ética como sendo o lema da organização.

Aytward et al. (2003), descreveram que a efetividade dos programas de educação no contexto dos cuidados de longa duração deve considerar fatores 
organizacionais e sistêmicos, como a forma e o modo na prestação da assistência. Modelos recentes, como o modelo de cuidado centrado no indivíduo, convidam ao compromisso de tornar as ILPIs espaços humanizados e acolhedores, fugindo aos modelos de instituições totais como nos manicômios, prisões e hospícios (FERRINI; FERRINI, 2008; TOLSON D.; ROLLAND Y; ANDRIEU, 2011).

Já utilizado na clínica e em cenários que envolvem processos de decisão, o modelo centrado no indivíduo considera a história, o contexto sociocultural, a oportunidade de escolha e a pessoa idosa como participante de seu processo de cuidado, com capacidade de escolher como deve ser cuidado (EKMAN et al., 2011). Nasce em contraposição a ênfase tecnicista voltada à segurança e à instituição e propõe uma mudança na cultura da assistência ao idoso, valorizando seus direitos e sua qualidade de vida (KANE, 2001). De acordo com esse modelo, o tédio, a solidão e a falta de auxílio são os principais causadores de sofrimento entre os residentes. Por essa razão, o cuidado deve seguir os princípios: amor para combater a solidão, atividades com propósito, diálogo permanente com os idosos, a fim de se conhecer seus desejos e expectativas e interações espontâneas. Esse modelo baseia-se na premissa de que o crescimento pessoal é uma experiência inerente à vida humana, acreditando que idosos e equipe crescem juntos, mesmo com as adversidades associadas ao processo de cuidar (FERRINI; FERRINI, 2008).

Baseia-se também nas seguintes premissas: estimular o contato com plantas, animais, crianças e outras pessoas; amar alguém é antídoto para a solidão; favorecer interações espontâneas e não preditivas; incentivar atividades significativas é essencial para a saúde humana; acreditar que uma comunidade centrada no idoso compartilha as decisões e convida o idoso a ser participante ativo de sua vida. $\mathrm{O}$ modelo de cuidado à pessoa contrapõe o modelo institucional, frequentemente disseminado entre profissionais e instituições sem considerar o idoso como participante central das suas intervenções. No entanto, para alcançar mudanças de paradigmas no processo do cuidado integral ao idoso institucionalizado é necessário percorrer um caminho bastante árduo e estar munido de competência para enfrentar os desafios (SALMAZO-SILVA; GUTIERREZ, 2013).

\section{Desafios}

Por ser relativamente recente a sistematização técnica e legal das ILPIs no Brasil, as políticas nessa área carecem de investimentos e de maior atenção, 
em especial na destinação de recursos, na qualificação dos profissionais e no investimento de cuidados de saúde mais efetivos, com enfoque no grau de dependência, saúde e bem-estar do idoso institucionalizado (FERRAND et al., 2014).

Com base nos dados do IPEA (CAMARANO, 2010) sobre o perfil da população idosa residente nas ILPIs, esses serviços apresentam uma dupla carga de necessidades: a social e a de saúde. A carga social é traduzida nas situações de violência e vulnerabilidade social e as dificuldades associadas à garantia do uso e acesso aos bens e serviços. No entanto, a carga de saúde relaciona-se às condições de saúde e fragilidade dessa população, requisitando o adequado gerenciamento das doenças crônicas; o reestabelecimento das condições de fragilidade; a reabilitação da condição física, motora e cognitivas; e o acesso aos serviços de saúde, em especial aos programas de assistência secundária em saúde (CAMARANO, 2010; CORTELLETTI; CASARA; HERÉDIA, 2010).

$\mathrm{O}$ arcabouço legal disponível até o momento destaca que o cuidado deve ser realizado pelas famílias e que as instituições asilares devem amparar os idosos nas suas necessidades sociais e de saúde, ofertando a atenção integral ao idoso e a inclusão social (BRASIL, 2003). Todavia, inexiste até o momento referência às responsabilidades do Estado no amparo às famílias que cuidam dos seus idosos. Se o envelhecimento é algo evidente, em especial com a sobrevida de idosos muito idosos e que segundo levantamentos recentes do IPEA são os mais dependentes e frágeis, nosso ponto de vista é que o desafio de cuidar desses idosos deve ser compartilhado e assumido pelo Estado (CAMARANO, 2010). As leis disponíveis, como a Política Nacional do Idoso (BRASIL, 1994) e a RDC n. 283 (ANVISA, 2005) não estabelecem o número mínimo de ILPIs e de serviços de atenção ao idoso que deveriam ser ofertados pelo Estado, deixando uma lacuna significativa na relação entre oferta e procura por esses serviços.

Por outro lado, quando os serviços de atenção existem, ainda apresentam dificuldades de funcionamento na sua totalidade por problemas estruturais, falta de formação de recursos humanos na área de Geriatria e Gerontologia, escassez de recursos materiais e excesso de demanda de atendimento (SALMAZO-SILVA; GUTIERREZ, 2013). Temos ainda, a fragmentação das necessidades, desarticulação das ações, barreiras de comunicação entre idosos, família e profissionais, e o acesso dificultado aos serviços (COSTA; CIOSAK, 2010).

Em estudo conduzido por Creutzberg et al. (2007), foi detectado desconhecimento mútuo entre ILPIs e o Sistema Único de Saúde (SUS), sendo necessária estimulação para um acoplamento estrutural mais sucedido. Políticas de atenção desenvolvidas em outros países como Espanha e Portugal provêm ajuda aos cuidadores familiares e uma oferta de serviços maiores, como centros dia e atendimento domiciliar. 
Infelizmente, no Brasil, as políticas de atenção ao envelhecimento avançam a passos lentos. Cabe destacar que a RDC n. 283 da ANVISA (2005) necessita de revisões quanto ao número de profissionais mínimos para idosos e quanto aos recursos humanos da área de saúde para atender aos idosos, como gerontólogos, fisioterapeutas, enfermeiros, terapeutas ocupacionais, psicólogos, nutricionistas, entre outros. Embora, as ILPIs sejam de caráter residencial e asilar, os residentes possuem demandas de saúde que não podem ser negligenciadas. Assim, torna-se fundamental uma revisão das diretrizes da RDC para considerar os fatores preditivos de institucionalização e associado a isso, a intervenção do Estado na efetivação de cuidados de qualidade. Ressaltamos que os cuidados de longa duração aos idosos carecem de muitos investimentos, em especial quanto à oferta e à variedade de programas. Para romper os estigmas da institucionalização se fazem necessários investimentos educacionais e de mudanças no cenário dos cuidados de longa duração a curto, médio e longo prazo.

Em meio aos vários desafios, destacamos: o desenvolvimento, a adaptação e a criação de protocolos de avaliação e de intervenção relativos aos cuidados de longa duração no Brasil; a implantação de processos educacionais para efetivação dos cuidados centrados no indivíduo, sendo esses agentes de mudanças nas políticas institucionais, governamentais e na formação de recursos humanos e técnicos adequados; a mudança na cultura de cuidados para focalizar o potencial e o idoso como participante e centro das intervenções; o investimento governamental e o desenvolvimento de políticas públicas, aliando-se ao progresso técnico-científico que pode trazer melhorias às instituições (Figura 1).

Conforme modelo (Figura 1), a educação seria o eixo condutor que leva ao aprimoramento do conhecimento técnico e das habilidades humanas no processo de cuidado. $\mathrm{O}$ aprimoramento dessas duas dimensões provocará mudanças na conformação das políticas públicas, legislação e cultura (o modo como o idoso institucionalizado é visto como sujeito de direitos). O aprimoramento das políticas vigentes agenciará a valorização das necessidades dos atendidos e em consequência, a melhor alocação de recursos, direcionados para a provisão dos cuidados e para ao aumento do arcabouço teórico sobre as ILPI. Em conjunto, todas essas ações convergem para mudanças organizacionais e na forma como os cuidados são ofertados, com a participação de atores como os profissionais gerontólogos, sociedade civil e de ações concretas que visam à melhoria dos cuidados. 
FIGURA 1 - MODELO HIPOTÉTICO DE COMO A EDUCAÇÃO PODE INSPIRAR MELHORIAS NOS CUIDADOS DE LONGA DURAÇÃO

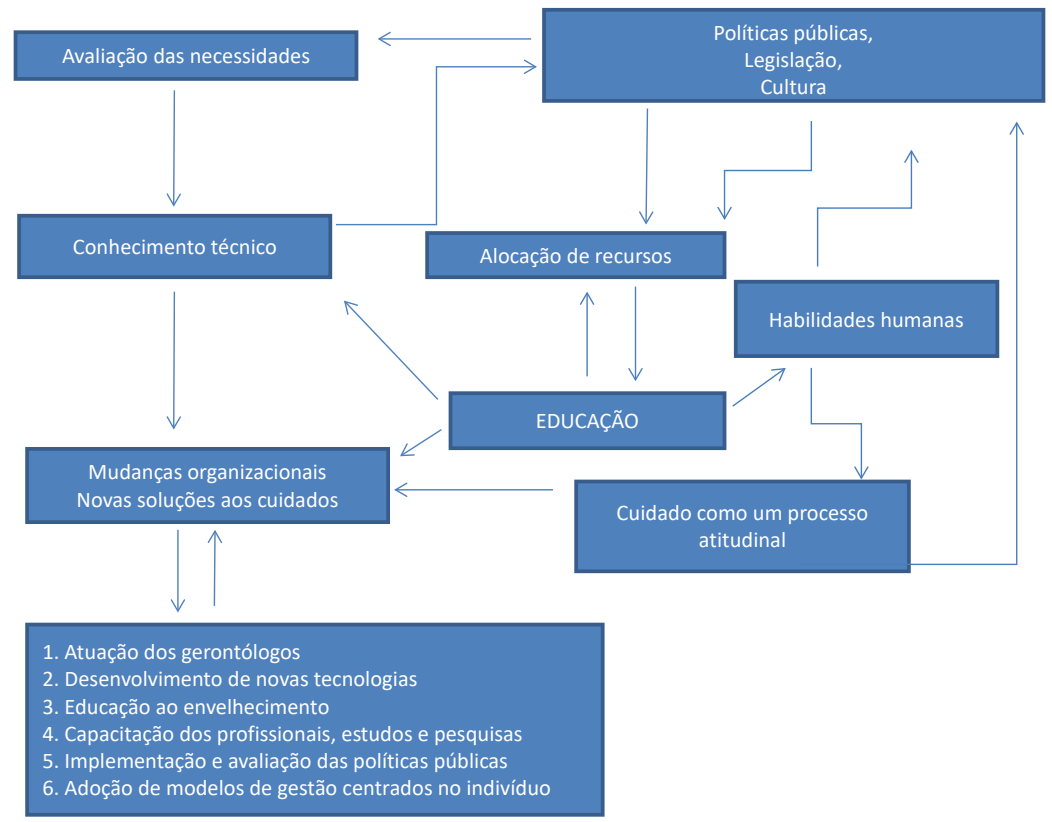

FONTE: Salmazo, Silva et al. (2017).

\section{Considerações finais}

Nessa abordagem, acredita-se que as mudanças na conformação dos cuidados exigem respostas que são pautadas na educação técnica e humana. Essas mudanças englobam dimensões de microgestão, voltadas ao atendimento direto ao idoso; mesogestão, direcionadas aos modelos organizacionais e a macrogestão, focalizando as políticas públicas.

Salienta-se que no Brasil é extremamente necessário colocar em pauta a qualidade dos serviços prestados aos residentes das ILPIs, proporcionando-lhes assim, dignidade na última fase do seu ciclo de vida, no entanto, isso só será possível se estiver fundamentada em um processo de educação totalmente reformulado e capaz de proporcionar ao profissional subsídios para que possa assistir os idosos na sua integralidade. 


\section{REFERÊNCIAS}

AGÊNCIA NACIONAL DE VIGILÂNCIA SANITÁRIA. Diretoria Colegiada. Resolução Anvisa/DC n.283, de 26 de dezembro de 2005, Diário Oficial da União, Poder Executivo, Brasília, DF, 27 set. 2005, Seção 1, p. 58-59.

ALCÂNTARA, A. O. Velhos institucionalizados e família: entre abafos e desabafos. Campinas/SP: Alínea, 2009.

AYTWARD, S.; STOLEE, P.; KEAT, N.; JOHNCOX, V. Effectiveness of continuing education in Long-term care: A literature review. The Gerontologist, v. 43, p. 259-271, 2003.

BALTES, M. The many faces of dependency in old age. Nova York: Cambridge University Press, 1996.

BRANDÃO, H. P; GUIMARÃES, T. A. Gestão de competência e gestão de Desempenho: tecnologias distintas ou instrumentos de um mesmo construto? Revista de Administração de Empresas, São Paulo, v. 41, n. 1, p. 8-15, jan./mar. 2001.

BRASIL. Política Nacional do Idoso. Lei n. 8.842, de 4 de janeiro de 1994. Dispõe sobre a política nacional do idoso, cria o Conselho Nacional do Idoso e dá outras providências. Brasília, 1994.

BRASIL. Estatuto do Idoso. Lei n. 10.741, de 1. de outubro de 2003. Dispõe sobre o Estatuto do Idoso e dá outras providências. Disponível em: $<$ http://www.planalto.gov. br/ccivil_03/leis/2003/L10.741.htm>. Acesso em: 13 jul. 2017.

BORN, T., BOECHAT, N.S. A qualidade dos cuidados ao idoso institucionalizado. In: EKMAN, I. et al. Person-centered care - Ready for prime Time. European Journal of Cardiovascular Nursing, London, v. 10, n. 4, p. 248-251, dez. 2011.

BROWNIE, S.; HORSTMANSHOF, L. The management of loneliness in aged care residents: An Important therapeutic target for gerontological nursing. Geriatric Nursing, New York, v. 32, n. 5, p. 318-325, set./out. 2011.

CAMARANO, A. A. (Org.) Cuidados de Longa Duração para a população idosa: um novo risco social a ser assumido? Rio de Janeiro: IPEA, 2010.

CAMARANO, A. A., KANSO, S. Envelhecimento da população brasileira: uma contribuição demográfica. In: FREITAS, E. V.; PY, L.; CANÇADO, F. A. X.; DOLL, J., (Org.). Tratado de Geriatria e Gerontologia. 3. ed. Rio de Janeiro: Guanabara Koogan, 2011. p. 1-17.

CAMARANO, A. A.; KANSO, S.; MELLO, J. L.; CARVALHO, D.F. As Instituições de Longa Permanência para Idosos no Brasil. In: CAMARANO, A. A. (Org.) Cuidados de Longa Duração para a população idosa: um novo risco social a ser assumido? Rio de Janeiro: IPEA, 2010, cap.7, p. 187-211.

COOPER, C.; SELWOOD, A.; LIVINGSTON, G. The prevalence of elder abuse and neglect: a systematic review. Age and Ageing, London, v. 37, n.2, p.151-160, mar. 2008. 
CORNALLY, N.; MCGLADE, C.; WEATHERS, E.; DALY, E.; FITZGERALD, C.; O'CAIOIMH, R.; COFFEY, A.; MOLLOY, D.W. Evaluating the systematic implementation of the ' Let me Decide' advance care planning programme in long term care through focus groups: staff perspectives. BMC Palliative Care, London, v. 3, 14:55, nov. 2015.

CORTELLETTI, I. A.; CASARA, M. B.; HERÉDIA, V. B. M. Idoso asilado: um estudo gerontológico. 2. ed. Caxias do Sul, RS: EDIPUCRS, 2010.

COSTA, M. F. B. N. A.; CIOSAK, S.I. Atenção integral na saúde do idoso do Programa da Saúde da Família: visão dos profissionais de saúde. Revista da Escola de Enfermagem da USP, São Paulo, v. 44, n. 2, p. 437-444, jun. 2010.

CREUTZBERG, M.; GONÇALVES, L. H. T.; SOBOTTKA, E. A; OJEDA, B.S. Longterm care institutions for elders and the health system. Rev. Latino-Am. Enfermagem, Ribeirão Preto, v. 15, n. 6, p. 1144-1149, nov./dez. 2007.

FERRAND, C. et al. Psychological need satisfaction and well-being in adults aged 80 years and older living in residential homes: Using a self-determination theory perspective. Journal of Aging Studies, v. 30, p. 104-111, 2014.

FERRINI, A. F.; FERRINI, R. L. Health in the later years. 4. ed. Boston: The McGrawHill Companies, Inc., 2008.

FONTES, A. P.; FORTES-BURGOS, A. C. G.; MELLO, D. M.; PEREIRA, A. A.; NERI, A. L. Arranjos domiciliares, expectativa de cuidado, suporte social percebido e satisfação com as relações sociais. In: NERI, A. L.; GUARIETO, M. E. (Org.). Fragilidade, saúde e bem-estar em idosos. Dados do Estudo Fibra Campinas. Campinas/SP: Alínea, 2011. p. 55-74.

FREITAS, E. V. et al. (Org.). Tratado de Geriatria e Gerontologia. 3. ed. Rio de Janeiro: Guanabara Koogan, 2011. p. 1299-1309.

GORZONI, M. L. (Org). Tratado de Geriatria e Gerontologia. 3. ed. Rio de Janeiro: Guanabara Koogan, 2011. p. 1-1741.

GUTIERREZ, B. A. O.; SALMAZO-SILVA, H.; SHIMIZU, H. E. Aspectos biopsicossociais e a complexidade assistencial de idosos hospitalizados. Acta Paulista de Enfermagem, São Paulo, v. 27, n. 5, p. 427-433, set./out. 2014.

HUSEBO, B. S.; FLO, E.; AARSLAND, D.; SELBAEK, G.; TESTAD, I.; GULLA, C.; AASMUL, I.; BALLARD, C. COSMOS - improving the quality of life in nursing home patients: protocol for an effectiveness-implementation cluster randomized clinical hybrid trial. Implementation Science, London, v. 10, p. 131, set. 2015.

KANE, R. A. Long-term care and a good quality of life: Bringing them close together. The Gerontologist, v. 41, p. 293-304, 2001.

LOW, L. F.; FLETCHER, J.; GOODENOUGH, B.; JEON, Y. H.; ETHERTON-BEER, C.; MACANDREW, M.; BEATTIE, E. A systematic review of interventions to change staff care practices in order to improve resident outcomes in Nursing Homes. Plos One, San Francisco, v. 10, e0140711, nov. 2015. 
MORLEY, R. et al. International Survey of Nursing Home Research Priorities. JAMDA, Columbia, v. 15, n. 5, p. 309-312, maio 2014.

NERI, A. Atitudes em relação à velhice: questões científicas e políticas. In: FREITAS, E. V. et al. (Org.). Tratado de Geriatria e Gerontologia. 2. ed. Rio de Janeiro: Guanabara Koogan, 2006.

PAVARINI, S. C. I. Dependência comportamental na velhice: uma análise do cuidado prestado ao idoso institucionalizado. 1996. 230 f. Tese (Doutorado em Educação) - Faculdade de Educação da Universidade Estadual de Campinas, Campinas, São Paulo, 1996.

PUTNAM, M.; TANG, F.; BROOKS-DANSO, A.; MORROW-HOWELL. N. Professional's beliefs about Nursing Home regulations in Missouri. Journal of Applied Gerontology, v. 28, p. 290-304, 2007.

SANTOS, C. C. N.; SANTOS, C. C. N.; SALMAZO-SILVA, H.; GUTIERREZ, B. A. O. Os cuidados de longa duração e a percepção de idosos institucionalizados sobre velhice, velhice bem-sucedida e qualidade da atenção. Revista Kairós Gerontologia, v. 20, p. 151-178, 2017.

SALMAZO-SILVA, H.; GUTIERREZ, B. A. O. Cuidados de longa duração na velhice: desafios para o cuidado centrado no indivíduo. A Terceira Idade, São Paulo, v. 24, n. 57, p.7-17, jul.2013.

SALMAZO-SILVA, H.; SILVEIRA, M. H.; FREITAS, T. H. S. Práticas Gerontológicas e os desafios da Gerontologia como ciência e profissão. In: CARVALHO, C. M. R. G.; ARAÚJO, L. F. (Org.). Envelhecimento e práticas gerontológicas. Curitiba: CRV Editora, 2017. v. 1.p. 169-188.

TANAKA, K. et al. Burnout of Long-term care facility employees: Relationship with employees' expressed emotion toward patients. International Journal of Gerontology, v. 9, p. 161-165, 2015.

THEURER, K.; MORTENSON, W. B.; STONE, R.; SUTO, M.; TIMONEN, V.; ROZANOVA, J. The need for social revolution in residential care. Journal of Aging Studies, v. 35, p. 201-210, dez. 2015.

TOLSON, D.; ROLLAND, Y.; ANDRIEU, S. International Association of Gerontology and Geriatrics: A global agenda for clinical research and quality of care in nursing homes. J. Am. Med. Dir. Assoc., United States, v. 2, n. 3, p. 184-189, mar. 2011.

TU, Y-C.; WANG, R.; YEH, S. Relationship between perceived empowerment care and quality of life among elderly residents within nursing homes in Taiwan: A questionnaire survey. International Journal of Nursing Studies, New York, v. 43, n. 6, p. 673-680, ago. 2006.

Texto recebido em 20 de julho de 2017 Texto aprovado em 03 de dezembro de 2017. 